\title{
Transient Receptor Potential Channel, Vanilloid 5, Induces Chondrocyte Apoptosis in a Rat Osteoarthritis Model Through the Mediation of $\mathrm{Ca}^{2+}$ Influx
}

\author{
Yingliang Weia Dianbin Zheng ${ }^{b} \quad$ Xiaocheng Guo ${ }^{b}$ Min Zhao ${ }^{c}$ Linlin Gaoc \\ Lunhao Baib \\ aDepartment of Orthopedic Surgery, ShengJing Hospital, China Medical University, ShenYang, \\ bDepartment of Orthopedic Surgery, ShengJing Hospital, China Medical University, ShenYang, 'Medical \\ Research Center, ShengJing Hospital, China Medical University, ShenYang, China
}

\author{
Key Words \\ Trpv5 $\bullet \mathrm{Ca}^{2+} \cdot$ Calmodulin $\bullet$ Chondrocyte apoptosis $\bullet$ Osteoarthritis
}

\begin{abstract}
Background/Aims: Chondrocyte apoptosis is the most common pathological feature in cartilage in osteoarthritis (OA). Transient receptor potential channel vanilloid 5 (TRPV5) is important in regulating calcium ion $\left(\mathrm{Ca}^{2+}\right)$ influx. Accumulating evidences suggest that $\mathrm{Ca}^{2+}$ is a major intracellular second messenger that can trigger cell apoptosis. Therefore, we investigate the potential role of TRPV5 in mediating $\mathrm{Ca}^{2+}$ influx to promote chondrocyte apoptosis in OA. Methods: The monoiodoacetic acid (MIA)-induced rat OA model was assessed by macroscopic and radiographic analyses. Calmodulin protein immunolocalization was detected by immunohistochemistry. The mRNA and protein level of TRPV5, calmodulin and cleaved caspase- 8 in articular cartilage were assessed by real time polymerase chain reaction and western blotting. Primary chondrocytes were isolated and cultured in vitro. TRPV5 small interfering RNA was used to silence TRPV5 in chondrocytes. Then, calmodulin and cleaved caspase- 8 were immunolocalized by immunofluorescence in chondrocyte. Fluo-4AM staining was used to assess intracellular $\mathrm{Ca}^{2+}$ to reflect TRPV5 function of mediation $\mathrm{Ca}^{2+}$ influx. Annexin $\mathrm{V}$-fluorescein isothiocyanatepropidium iodide flow cytometric analysis was performed to determine chondrocytes apoptosis. Western blotting techniques were used to measure the apoptosis-related proteins in chondrocyte level. Results: Here, we reported TRPV 5 was up-regulated in MIA-induced OA articular cartilage. Ruthenium red (a TRPV5 inhibitor) can relieve progression of joint destruction in vivo which promoted us to demonstrate the effect of TRPV5 in OA. We found that TRPV5 had a specific role in mediating extracellular $\mathrm{Ca}^{2+}$ influx leading to chondrocytes apoptosis in vitro. The apoptotic effect was inhibited even reversed by silencing TRPV5. Furthermore, we found that the increase $\mathrm{Ca}^{2+}$ influx triggered apoptosis
\end{abstract}


by up-regulating the protein of death-associated protein, FAS-associated death domain, cleaved caspase-8, cleaved caspase-3, cleaved caspase-6, and cleaved caspase-7, and the upregulated proteins were abolished by silencing TRPV5 or 1, 2-bis-(o-Aminophenoxy)-ethane$\mathrm{N}, \mathrm{N}, \mathrm{N}^{\prime}, \mathrm{N}^{\prime}$-tetraacetic acid, tetraacetoxymethyl ester (a $\mathrm{Ca}^{2+}$ chelating agent). Conclusion: The up-regulated TRPV 5 could used be as an initiating factor that induces extrinsic chondrocyte apoptosis via the mediation of $\mathrm{Ca}^{2+}$ influx. These findings suggested TRPV5 could be an intriguing mediator for drug target in $\mathrm{OA}$.

\section{Introduction}

Osteoarthritis (OA) can cause pain, stiffness, swelling, and loss of function in the joints, which greatly affects quality of life in the elderly population. In the Global Burden of Disease 2010 study, knee OA was ranked the $38^{\text {th }}$ highest of 291 conditions contributing to global disability [1]. Pathologically, OA is characterized by the progressive degeneration of articular cartilage [2]. Articular cartilage depends solely on its resident cells, chondrocytes, to maintain the extracellular matrix. The cartilage is often accompanied by lacunar emptying, which is evidence that chondrocyte apoptosis is a central feature in OA progression [3]. Thus, in the progression of cartilage degeneration, finding a cause for chondrocyte apoptosis during $\mathrm{OA}$ may become a potential strategy against $\mathrm{OA}$ disease.

$\mathrm{Ca}^{2+}$ is a major intracellular second messenger and a key regulator of cell survival. The disruption of $\mathrm{Ca}^{2+}$ homeostasis due to its sustained elevation in the cytoplasm can trigger apoptosis [4]. Our recent study described the role of $\mathrm{Ca}^{2+}$ influx through $\mathrm{Ca}^{2+}$-selective channels, such as transient receptor potential channel vanilloid 5 (TRPV5), and that the increase $\mathrm{Ca}^{2+}$ in the intracellular space can inhibit chondrocyte autophagy in OA by activating calmodulin-dependent protein kinase 2 (CAMK II) [5]. Transient receptor potential channel vanilloid 5 (TRPV5) is a member of the TRPV subfamily, and functions as a facilitative $\mathrm{Ca}^{2+}$ transporter [6]. TRPV5 is particularly important in regulating Ca2+ influx to maintain Ca2+ homeostasis [7]. Loss of TRPV5 function results in abnormal ionocyte proliferation and increased colon cancer risk [8]. TRPV5 may contribute to the process of estrogeninhibited osteoclastogenesis and bone resorption activity by mediating extracellular Ca2+ [9]. The increase in intracellular $\mathrm{Ca} 2+$ levels can result activation of the $\mathrm{Ca} 2+$ sensor protein calmodulin and combined target proteins to form the Ca2+/calmodulin complex [10]. $\mathrm{Ca}^{2+}$ / calmodulin complexes can participate in the extrinsic apoptotic pathway by promoting the recruitment of FAS-associated death domain (FADD) [11]. Activated FADD initiates caspase-8 activation, which in turn is released into the cytoplasm and initiates further downstream caspase cascades, including caspase-3, 6, and 7 [12]. However, the mechanism of TRPV5mediated $\mathrm{Ca}^{2+}$ influx in the activation of the extrinsic apoptotic pathway in chondrocytes requires further investigation.

The purpose of this study is to reveal the potential regulatory mechanism of TRPV5 in mediating $\mathrm{Ca}^{2+}$ influx and initiating chondrocyte apoptosis in an MIA-induced rat OA model. Therefore, TRPV 5 could be a potential therapeutic target for OA treatment.

\section{Materials and Methods}

\section{Animals and Development of MIA-Induced Rat OA Models}

Male Sprague-Dawley Rats (2 months old, 220-230 g in weight) were used. Experimental animals groups flowchart was arranged in Fig. 1. All rats were housed in groups of five per cage under standard laboratory conditions with free access to food and water, and a constant room temperature $\left(22^{\circ} \mathrm{C}\right)$ and humidity ( $45 \%$ to 50\%). Monosodium iodoacetate (MIA, Sigma USA) was dissolved in sterile saline $(0.9 \%$ $\mathrm{NaCl}$ ). Rats were randomly divided into groups as described below. Rats were given an intra-articular injection of MIA and ruthenium red through the infra-patella ligament of both knees, at a dose of $1 \mathrm{mg}$ in 


\section{Cellular Physiology Cell Physiol Biochem 2018;46:687-698 and Biochemistry \begin{tabular}{c|c} 
DOI: 10.1159/000488725 & $\begin{array}{l}\text { O 2018 The Author(s). Published by S. Karger AG, Basel } \\
\text { www.karger.com/cpb }\end{array}$
\end{tabular}

$50 \mu$ sterile saline and control (normal) animals were given an intra-articular injection of equivolume sterile saline.

\section{Macroscopic Analysis}

Joint space was monitored using the digital X-ray (MX-20, Faxitron X-Ray Corp., Wheeling, IL, US). X-rays were graded as follows: $0=$ normal appearance; 1 = slight narrowing of the joint

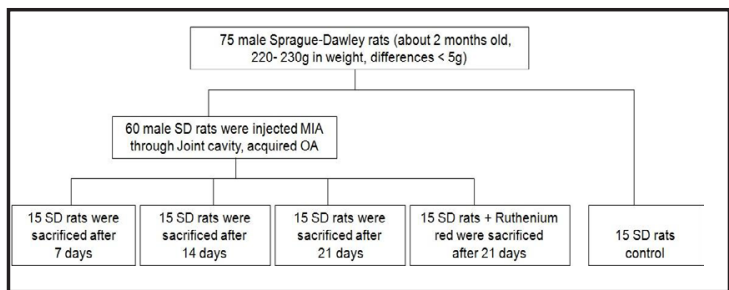

Fig. 1. Experimental animals groups flowchart. space; 2 = narrowing of the joint space but with no osteophytes; 3 = severe narrowing of the joint space with some osteophytes; $4=$ severe narrowing of the joint space with many osteophytes. The articular appearance of macroscopic lesions was graded as follows: 0 = normal appearance; 1 = slight yellowish discoloration of the chondral surface; 2 = small cartilage erosions in load-bearing areas; 3 = large erosions extending down to the subchondral bone; 4 = large erosions with large areas of subchondral bone exposure. Each of the chondral compartments (the femoral condyles, the tibial plateaus, the patella, and the femoral groove) were examined. All samples were measured by three assessors who were blinded to the induction procedure.

RT-PCR

The RNA of chondrocytes was isolated using a Trizol reagent (Invitrogen, USA). According to the manufacturer's instructions, RNA was reverse-transcribed into complementary DNA using the PrimeScript RT reagent kit (Takara RR036A, Shiga, Japan). RNA expression of TRPV5 was evaluated by real-time PCR using SYBR® GreenER ${ }^{\mathrm{TM}}$ SuperMix (Takara, Shiga, Japan) with the specific primer for TRPV5 (Fw: 5'-CTGCCTGTGTGGGTAGTGAG-3' and Rv: 5'-GGTGAGTCCTTGGTTGTTGG-3'), calmodulin (Fw: 5'-GGCATCCTGCTTTAGCCTGAG-3' and RV: 5'-ACATGCTATCCCTCTCGTGTGA-3'), cleaved caspase-8 (Fw: 5'-ACTGGCTGCCCTCAAGTTCCTGTGC-3' and RV:5'-TCCCTCACCATTTCCTCTGGGCTGC-3'), $\beta$-actin (Fw: 5'-CAGCCTTCCTTCCTGGGTATG-3' and Rv: 5'-TAGAGCCACCAATCCACACA-3') and an ABI Prism 7500 sequence detection system (Applied Bio-systems, Foster City, CA, USA). The fold changes in gene expression were calculated using the following formula: $2^{-\Delta c t}$ where $\Delta c t$ is the difference of the threshold cycle of the sample.

\section{Immunohistochemistry (IHC)}

The resected knee samples were fixed in neutral formalin-buffered solution for 2 weeks, then decalcified in EDTA decalcification liquid for 3 weeks. Samples were embedded in paraffin and cut into 4- $\mu$ m tissue sections. After antigen retrieval and blocking endogenous peroxidase activity, sections were then incubated with anti-calmodulin antibody (Abcam, Cambridge, UK; 1:200 dilution) at $4^{\circ} \mathrm{C}$ overnight. Then the secondary antibody (Zhongshanjinqiao, Beijing, China) was applied for $30 \mathrm{~min}$ at room temperature. Staining was detected with DAB (3, 3'-diaminobenzidine tetrahydrochloride). Sections were next counterstained with hematoxylin for 4 minutes to stain the nucleus and then dehydrated with ascending concentrations of ethanol solution, cleared with xylene and mounted with a coverslip.

\section{Isolation, culture and identification of rat primary chondrocytes}

Primary chondrocytes were isolated from rats as described [5]. Fresh medium was replaced every 2 days and chondrocytes reached approximately $80 \%$ confluence by days 4-5 as the $\mathrm{P}_{0}$ generation. Confluence chondrocytes were then detatched with trypsin for subculture continually as generations P1, P2, and P3. Cells were used for experiments within the $\mathrm{P}_{3}$ generation. Immunocytochemistry was performed to identify chondrocyte phenotypes. Monolayer cells were incubated with anti-type II collagen antibody (Abcam, Cambridge, UK; 1:400 dilution) at $4^{\circ} \mathrm{C}$ overnight. The secondary antibody was then applied for $30 \mathrm{~min}$ at room temperature. Staining was detected with DAB.

\section{Small interfering RNA transfection}

Chondrocytes were transfected at 60-70\% confluence using siRNA transfection reagent (sc-29528; Santa Cruz Biotechnology, Santa Cruz, CA, USA) with TRPV5 siRNA (sc-42676; Santa Cruz Biotechnology, Santa Cruz, CA, USA) diluted into siRNA transfection medium (sc-36868; Santa Cruz Biotechnology, Santa 


\section{Cellular Physiology Cell Physiol Biochem 2018;46:687-698 \begin{tabular}{l|l} 
DOI: 10.1159/000488725 & Ond 2018 The Author(s). Published by S. Karger AG, Basel \\
www.karger.com/cpb
\end{tabular} \\ Wei et al:: Transient Receptor Potential Channel, Vanilloid 5, Induces Chondrocyte Apoptosis}

Cruz, CA, USA) at a final concentration of $100 \mathrm{nM}$ according to the manufacturer's instructions. Chondrocytes that were transfected with scrambled control siRNA (sc-37007; Santa Cruz Biotechnology Santa Cruz, CA, USA) at a concentration of $100 \mathrm{nM}$ were served as the negative control. Cells were transfected for $72 \mathrm{~h}$ for the TRPV5 knockdown study prior to $\mathrm{Ca}^{2+}$ measurement, immunofluorescence and western blot analysis.

\section{Immunofluorescence staining}

Chondrocytes and chondrocytes transfected with TRPV5 or control siRNA were seeded at a density of $1 \times 10^{6}$ cells/well in complete growth medium in a 6 -well plate for $24 \mathrm{~h}$. The following day, cells were treated with 0-6 $\mu \mathrm{M}$ MIA for $12 \mathrm{~h}$, and one group was pre-incubated with $10 \mu \mathrm{M}$ ruthenium red for 30 minutes before $6 \mu \mathrm{M}$ MIA incubation. Each well was brought to a final volume of $2 \mathrm{ml}$ with complete growth medium. Treated cells were then fixed in neutral formalin-buffered solution for 30 minutes, washed three times with PBS and incubated with primary anti-calmodulin antibody (Abcam, Cambridge, UK; 1:200 dilution) and anti-cleaved caspase-8 (Abcam, Cambridge, UK; 1:400 dilution) antibodies overnight at $4^{\circ} \mathrm{C}$. Samples were then incubated with secondary antibody (Abcam, USA, 1:100 dilution) for $1 \mathrm{~h}$ at $37^{\circ} \mathrm{C}$. The chondrocyte nuclei were stained with DAPI (4',6-diamidino-2-phenylindole) for $5 \mathrm{~min}$. The stained chondrocytes were observed under a fluorescence microscope.

\section{Determination of intracellular $\mathrm{Ca}^{2+}$ by fluo-4AM staining}

Chondrocytes and chondrocytes transfected with TRPV5 or control siRNA were seeded at a density of $1 \times 106$ cells/well in complete growth medium in a 6 -well plate for $24 \mathrm{~h}$. The following day, cells were treated with 0-6 $\mu \mathrm{M}$ MIA for $12 \mathrm{~h}$, and two wells were pre-incubated with $10 \mu \mathrm{M}$ ruthenium red and 10 $\mu \mathrm{M}$ BAPTA-AM, respectively, for 30 minutes then washed with PBS before $6 \mu$ M MIA incubation. Each well was brought to a final volume of $2 \mathrm{ml}$ with complete growth medium. Treated cells were washed three times with D-Hanks balanced salt solution without Ca2+. Subsequently, cells were loaded with $2 \mu \mathrm{mol} / \mathrm{l}$ Fluo-4AM (Dojindo, Japan) for $30 \mathrm{~min}$ at $37^{\circ} \mathrm{C}$ in the dark, then washed twice with D-Hanks balanced salt solution without Ca2+ to remove extracellular Fluo-4/AM. Imaging was performed using an OLYMPUS IX71 inverted microscope and analyzed with Image-Pro Plus 6.0. The measured average fluorescence intensity of each cell in the field (F) was normalized with the non-specific background fluorescence (F0) to obtain the fluorescence intensity (F/F0) [13]. Statistical data are expressed as percentage variation of treatment group vs. control $(0 \mu \mathrm{M}$ MIA).

\section{Detection of apoptosis by flow cytometry}

MIA-induced chondrocyte apoptosis was detected using an annexin V-FITC/PI apoptosis detection kit (AD101; Dojindo, Japan). Chondrocytes were grouped as in determination of intracellular $\mathrm{Ca}^{2+}$ described above in determination of intracellular $\mathrm{Ca} 2+$ by fluo-4AM staining. Chondrocytes were washed twice with PBS containing 5\% FCS and resuspended in $500 \mu \mathrm{l}$ binding buffer provided in the detection kit, followed by incubation with $5 \mu \mathrm{l}$ annexin V-FITC and $5 \mu \mathrm{l}$ propidium iodide (PI) at room temperature for $15 \mathrm{~min}$ in the dark. Flow cytometry was performed using Cell Quest software (BD Biosciences, San Jose, CA, USA).

\section{Western- blotting}

Chondrocytes were grouped as in determination of intracellular $\mathrm{Ca}^{2+}$ described above. Western blotting was performed as previously described [5]. Blots were incubated with primary antibodies including TRPV5 (Abcam, USA, 1:2000 dilution), calmodulin (Abcam, USA, dilution 1:1000), DAP (Abcam, USA, dilution 1:2000), FADD (Abcam, USA, dilution 1:2000), cleaved-caspase-8 (Abcam, USA, dilution 1:5000), cleavedcaspase-3 (Abcam, USA, dilution 1:5000), cleaved-caspase-6 (Abcam, USA, dilution 1:5000), cleavedcaspase-7 (Abcam, USA, dilution 1:5000), and $\beta$-actin (Abcam, USA, dilution 1:10000).

\section{Statistical Analysis}

All experiments in this study were repeated three times. All data are expressed as mean \pm standard error of the mean (SEM). Differences between means were analyzed using one-way analysis of variance (ANOVA) with MIA injected different at times and at different concentrations as the independent factors. A paired $t$-test was applied to evaluate the differences between $6 \mu \mathrm{M}$ MIA appended different intervention treatment and $6 \mu \mathrm{M}$ MIA alone. All statistical analyses were performed using SPSS 17.0 (IBM, Armonk, NY, USA). Significance was set at $P=0.05$ for all statistical analyses.

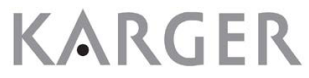




\section{Cellular Physiology and Biochemistry Published online: April05, $2018 \quad \begin{aligned} & \text { DOI: 10.1159/000488725 } 2018 \text { The Author(s). Published by S. Karger AG, Basel } \\ & \text { www.karger.com/cpb }\end{aligned}$}

Wei et al.: Transient Receptor Potential Channel, Vanilloid 5, Induces Chondrocyte Apoptosis

\section{Results}

Macroscopic and radiographic analyses and effect of the TRPV5 inhibitor, ruthenium red, on $O A$ progression in the MIA-induced rat OA model

Radiographic and macroscopic graphs with their evaluation scores of the knee joints were assessed. In the radiographic graph Fig. 2A, the surface of the knee joints was smooth in the normal (sterile saline) group. In contrast, obvious osteophytes as well as incomplete and thickened articular surfaces were observed in the OA group, particularly with the longest time of MIA injection. Regarding macroscopic evaluation of the OA group (Fig. 2B), the cartilage on the articular surface was thin and yellowish, with focal erosions of the tibial plateaus. Moreover, pathological features became increasingly severe as the MIA

Fig. 2. Macroscopic and radiographic analyses and effect of the TRPV5 inhibitor (Ruthenium red) in the MIA-induced rat OA model. Flowchart representing the groups of experimental animals (A) Macroscopic photographs of tibial plateaus of the rat knee joints (B) X-ray photographs of the total knee joints (C) Radiographic scores measuring joint destruction (D) Macroscopic scores measuring joint destruction. Data are presented as mean \pm SEM ( $\mathrm{n}=3$ ). ${ }^{*} \mathrm{P}<0.05$ vs. untreated control; ${ }^{\#} \mathrm{P}<0.05$ between MIA 21 day and MIA + ruthenium red 21 day treatment groups; ${ }^{* *} \mathrm{P}<0.05$ vs. untreated control; ${ }^{\# P} \mathrm{P}<0.05$ between MIA 21 day and MIA + ruthenium red 21 day groups. MIA, monosodium iodoacetate.

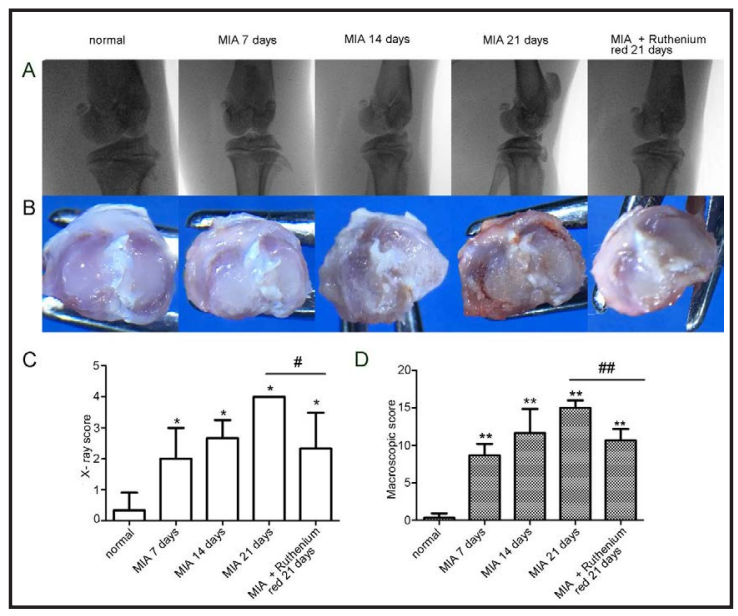

Fig. 3. TRPV5 expression is upregulated in the articular cartilage of MIA-induced OA and, which positively correlates with protein expression of calmodulin and the apoptosis related protein cleaved caspase-8 in OA articular cartilage. (A) Reverse transcription polymerase chain reaction (RT-PCR) showing the level of TRPV5 mRNA in OA from different MIA-stimulated groups. ${ }^{*} \mathrm{P}<0.05$ vs. untreated control, ${ }^{\#} \mathrm{P}>0.05$ between MIA 21 day and MIA + ruthenium red 21 day treatment groups; (B) RT-PCR showing the level of calmodulin mRNA in $\mathrm{OA}$ from different stimulated groups. ${ }^{*} \mathrm{P}<0.05$ vs. untreated control; ${ }^{\#} \mathrm{P}<0.05$ between MIA 21 day and MIA + ruthenium red 21 day treatment groups. (C) RT-PCR showing the level of cleaved caspase-8 mRNA in OA from different stimulated groups as compared to the normal group. ${ }^{*} \mathrm{P}<0.05$ vs. untreated control, " $\mathrm{P}<0.05$ between MIA 21 day and MIA + ruthenium red 21 day treatment groups. (D) TRPV5, calmodulin, cleaved caspase-8 protein expression from different stimulated groups at each time point as detected by western blotting. (E) A bar graph showing the levels of TRPV5,

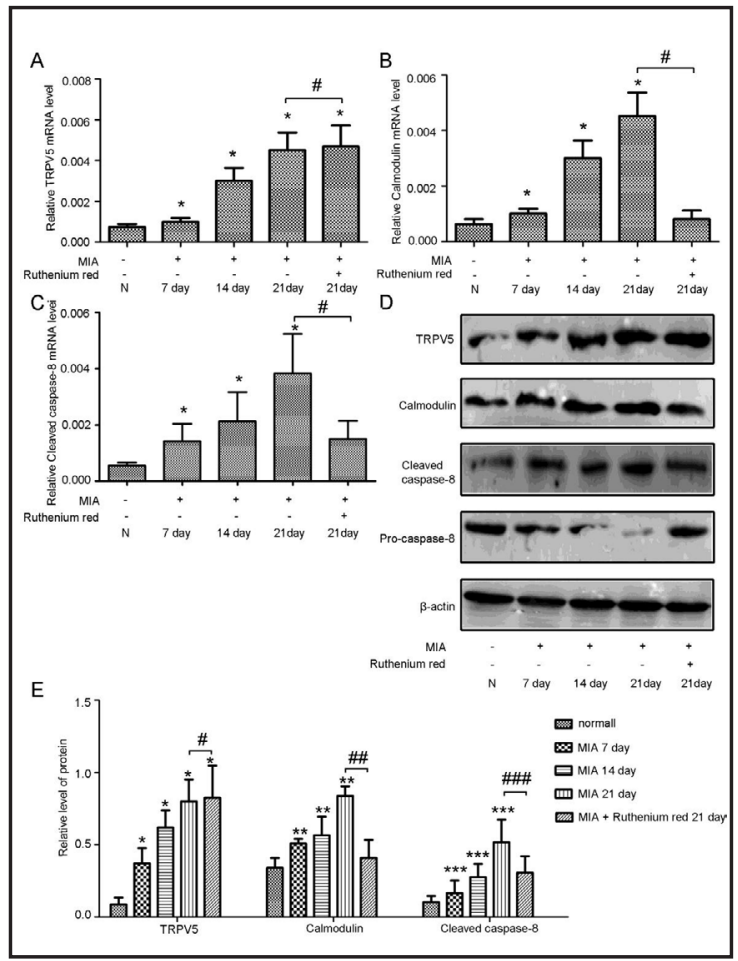
calmodulin and cleaved caspase- 8 proteins in various treatment groups. ${ }^{*} \mathrm{P}<0.05,{ }^{* *} \mathrm{P}<0.05$, ${ }^{* * *} \mathrm{P}<0.05$ vs. untreated control, ${ }^{\#} \mathrm{P}>0.05,{ }^{* \#} \mathrm{P}<0.05,{ }^{\# \# \#} \mathrm{P}<0.05$ between MIA 21 day and the MIA + ruthenium red 21 day treatment groups. 
injection time lengthened. The gradual deterioration of cartilage successfully simulated the progressive aggravation characteristic of OA over time. However, the MIA + ruthenium red group had markedly reduced pathological processes in both radiographic and macroscopic imagery. The evaluation scores at varying time points of the MIA group were significantly different, but consistent with the pathologic level of $\mathrm{OA}$ in radiographic and macroscopic graphs (Fig. 2C, 2D). These results suggest that, in articular chondrocytes, inhibition of TRPV5 using ruthenium red may be protective against the development of $\mathrm{OA}$, and that TRPV5 may participate in progressive cartilage destruction during OA.

\section{TRPV5 expression was confirmed in normal articular cartilage and TRPV5 was upregulated in $O A$ articular cartilage}

TRPV5 mRNA (Fig. 3A) was detected by RT-PCR and TRPV5 protein was identified by western blotting in articular cartilage. TRPV5 was weakly expressed in normal (sterile saline) cartilage. Accordingly, high TRPV5 mRNA expression was detected in the articular cartilage of the most severe OA (MIA 21 days; Fig. 3D), which is consistent with results obtained via western blotting. TRPV5 levels were significantly greater in the OA cartilage, and the degree of up-regulation was positively correlated with the degree of $\mathrm{OA}$ lesions ( Fig. 3E, $P<0.05$ ).

Upregulated expres-
sion of calmodulin and
the apoptosis maker
cleaved caspase-8 cor-
relates positively with
the progression of os-
teoarthritis induced by
MIA and
Calmodulin cleaved caspase-8 mRNA (Fig. 3B; 3C) were weakly expressed in normal (sterile saline) cartilage, but high expression was detected in the most severe OA articular cartilage (MIA 21 days). Calmodulin and apoptosis marker cleaved-caspase 8 protein levels as detected by western blotting (Fig. 3D) were consistent with RT-PCR results. Calmodulin protein was immunolocalized in articular cartilage by immunohistochemistry. In normal cartilage, its staining is very light (sterile saline; Fig. 4A). Mild staining of calmodulin was observed in moderately degenerative articular cartilage (MIA 7

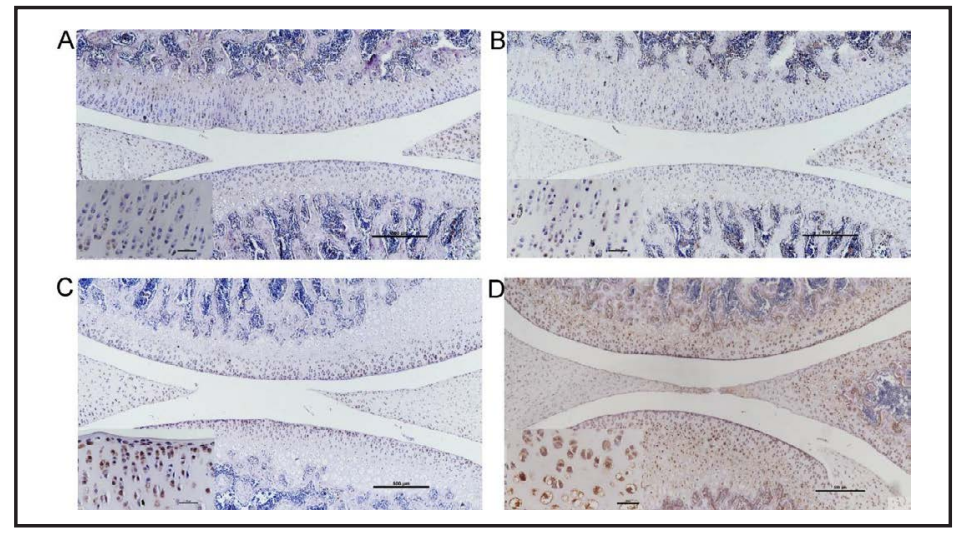

Fig. 4. Calmodulin protein in articular cartilage was immunolocalized via immunohistochemistry, and positively correlates with the progression of osteoarthritis in an MIA-induced rat OA model. Brown staining indicates specific calmodulin protein, and blue staining indicates the nucleus. The distribution of brown staining is positively correlated with calmodulin protein expression. (A) Normal rat knee joints (control group). (B) Rat knee joint section on MIA-induced OA for 7 days. (C) Rat knee joint section on MIA-induced OA for 14 days. (D) Rat knee joint section on MIA-induced OA for 21 days. (original magnification $\times 40$ and zoom-in magnification $\times 200$ ).

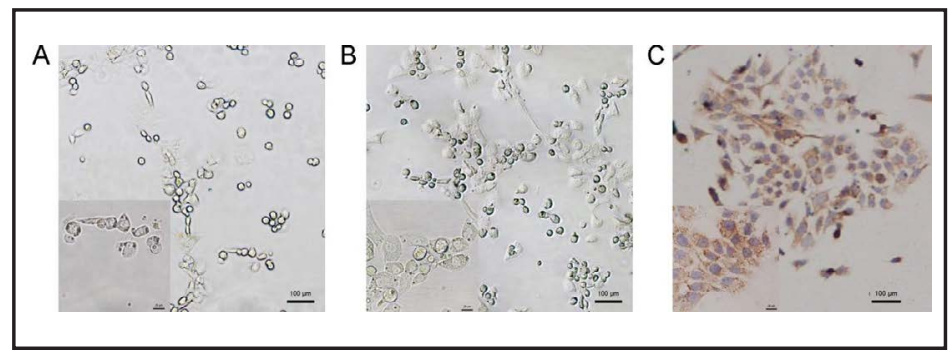

Fig. 5. Isolation, culture and identification of rat primary chondrocytes. (A) Primary chondrocyte culture after (A) 36h, (B) 72h. (C) Primary chondrocytes were stained with anti-collagen II to indicate functional chondrocytes (original magnification $\times 40$ and zoom-in magnification $\times 400$ ). 
Fig. 6. TRPV5 knockdown identification in rat primary chondrocytes. (A) Real-time polymerase chain reaction (RT-PCR) showed reduced TRPV5 mRNA levels in the TRPV5 siRNA group as compared to the normal group, ${ }^{*} \mathrm{P}<0.05$; as compared to the TRPV5 scrambled siRNA group, ${ }^{* *} \mathrm{P}<0.05$. (b) Western blotting shows reduced TRPV5 protein expression in the TRPV5 siRNA group. (C) A bar graph shows the level of reduced level of TRPV5 protein in the TRPV5 siRNA group as compared to

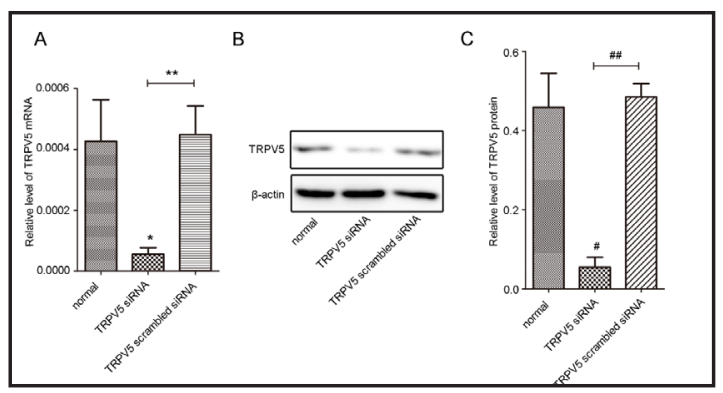
the normal group, ${ }^{\#} \mathrm{P}<0.05$; and as compared to the TRPV5 scrambled siRNA group, ${ }^{\#} \mathrm{P}<0.05$.

Fig. 7. Calmodulin expression and Cleaved caspase-8 in different chondrocyte treatment groups. Expression of (A) calmodulin (red) and (B) cleaved caspase-8 (red) was determined by immunofluorescence staining (original magnification $\times 200$ ). MIA, monosodium iodoacetate; RR, ruthenium red; DAPI, blue nuclear stain.

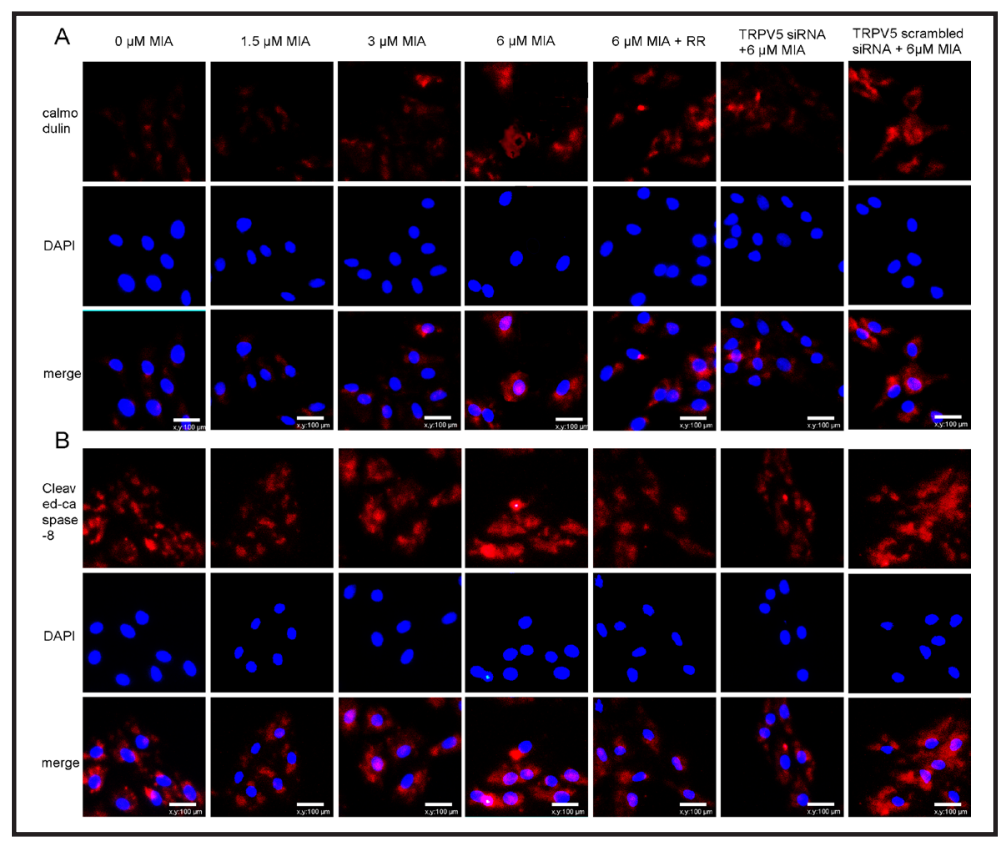

days; Fig. 4B), while staining became progressively higher with increased OA severity (MIA 14 and 21 days; Fig. 4C and 3D respectively), which suggests that calmodulin expression was up-regulated gradually with the OA progression development. In summary, these results suggest up-regulated expression of calmodulin and cleaved caspase-8 correlate positively with the progression of osteoarthritis induced by MIA.

Morphological observation of primary rat chondrocytes, and calmodulin and cleaved caspase-8 expression in normal and MIA-stimulated chondrocytes

Chondrocyte morphology, as observed under the microscope, is rounded early after plating and becomes flat and polygonal shape by $72 \mathrm{~h}$ of growth (Fig. 5A, B). Primary chondrocytes were stained with anti-collagen II (Fig. 5C) for identification. In order to investigate the effect of TRPV5 in MIA-stimulated chondrocyte, Small interfering RNA transfection technique was used to knockdown TRPV5 in rat primary chondrocytes. The silenced levels of TRPV5 mRNA and TRPV5 protein were identified 72h later (Fig. 6). We observed calmodulin and cleaved caspase- 8 expression in chondrocytes in vitro by immunofluorescence staining. As shown in Fig. 7A and 7B, immunofluorescence staining of both calmodulin and cleaved caspase- 8 in the MIA-stimulated group gradually increased with increasing MIA concentrations. However, calmodulin and cleaved caspase-8 staining were diminished in the ruthenium red group and TRPV5 siRNA groups. Thus, calmodulin and cleaved caspase- 8 expression in chondrocytes may be linked to TRPV5 expression in experimental OA.

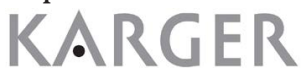


Fig. 8. Fluorescent images of rat primary chondrocytes labelled with the $\mathrm{Ca}^{2+}$ indicator dye Fluo4AM. (A) $\mathrm{Ca}^{2+}$ fluorescence relative intensity in different chondrocyte treatment groups (all photomicrographs are shown at $\times 200$ magnification). (B) Bar graph showing the level of relative fluorescent intensity in each group. ${ }^{*} \mathrm{P}<0.05 ; \quad * * \mathrm{P}<0.05 ; \quad * * * \mathrm{P}<0.05$; **** $\mathrm{P}<0.05 ; \quad$ *\# $\mathrm{P}<0.05 ; \quad \# \mathrm{P}<0.05$;

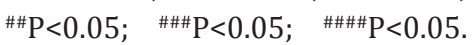
**\#\# $\mathrm{P}<0.05$. Each column represents mean \pm SEM $(n=3)$. MIA, monosodium iodoacetate; RR, ru-

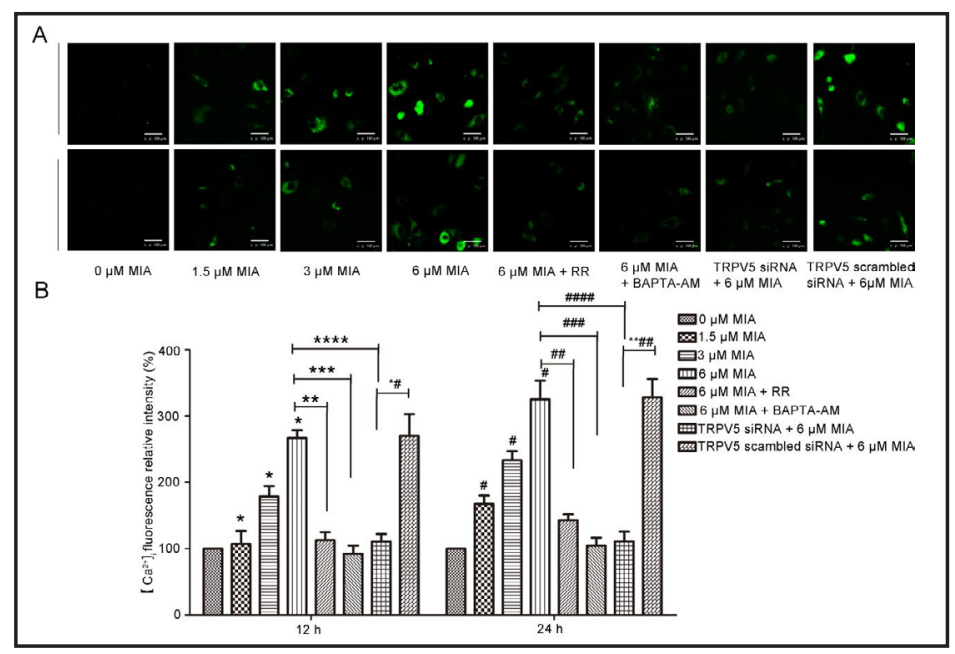
thenium red; TRPV5, transient receptor potential channel vanilloid 5; BAPTA-AM, 1, 2-bis-(o-Aminophenoxy)-ethane-N,N,N',N'-tetraacetic acid, tetraacetoxymethyl ester.

Fig. 9. Effects of TRPV5 on MIA-induced chondrocyte apoptosis. (A) Flow cytometry with Annexin V-FITC/PI staining of chondrocytes. (B) Bar graph showing the apoptosis rate of each group. ${ }^{*} \mathrm{P}<0.05$ difference vs. untreated group; \# $\mathrm{P}<0.05$; \#\# $\mathrm{P}<0.05$; \#\#\# $\mathrm{P}<0.05$ \#\#\#\#\# $\mathrm{P}<0.05$ difference vs. $6 \mu \mathrm{M}$ MIA treatment. Each column represents mean \pm SEM ( $\mathrm{n}=3$ ). MIA, monosodium iodoacetate; $\mathrm{RR}$, ruthenium red; TRPV5, transient receptor potential channel vanilloid 5; BAPTA-AM, 1, 2-bis-(o-Aminophenoxy)ethane-N,N,N',N'-tetraacetic acid, tetraacetoxymethyl ester.

TRPV5 can mediate calcium influx, which initiates chondro-

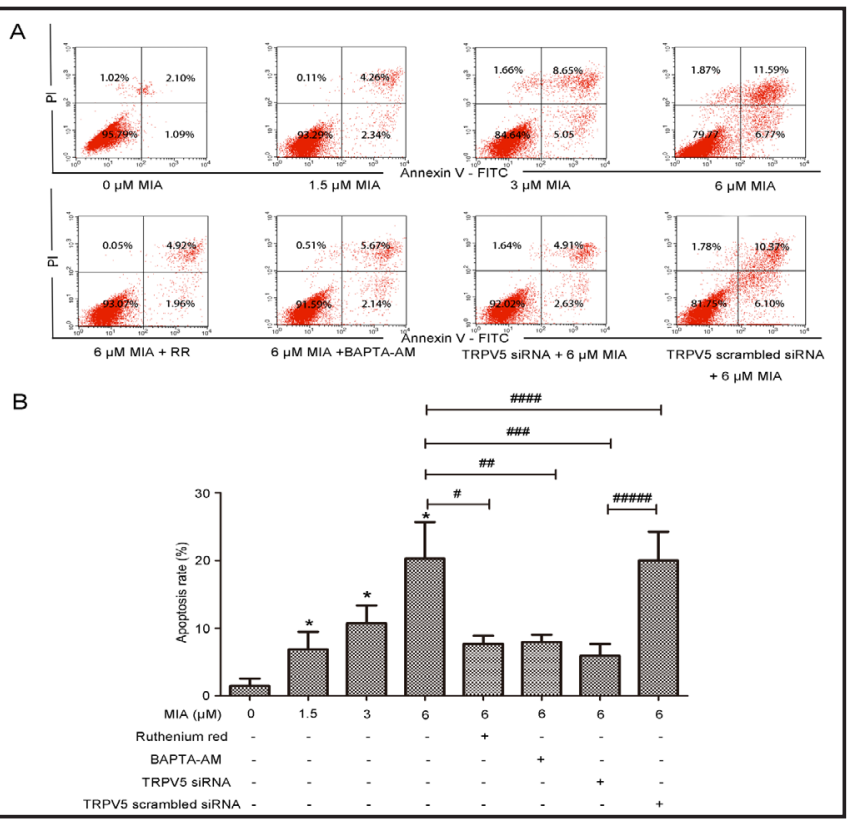
cyte apoptosis in MIA-stimulated chondrocytes

Calcium influx results show that the relative fluorescence intensity in the MIA-stimulated group gradually increases with increasing concentrations of MIA (0-6 $\mu \mathrm{M})$ for $12 \mathrm{~h}$ and 24h (Fig. 8A, 8B), while the fluorescence intensity was significantly reduced after treatment with ruthenium red $(10 \mu \mathrm{M})$ and the $\mathrm{Ca}^{2+}$ chelator BAPTA-AM $(10 \mu \mathrm{M})$. Additionally, fluorescence intensity was significantly reduced in chondrocytes transfected TRPV5 siRNA. These results indicate that TRPV5 may have a specific role in mediating extracellular $\mathrm{Ca}^{2+}$ influx.

Flow cytometric analysis results show that the percent of apoptotic cells significantly increases with increasing MIA concentrations, but is dramatically attenuated in the presence ruthenium red and BAPTA-AM or in chondrocytes transfected TRPV5 siRNA (Fig. 9https:// www.ncbi.nlm.nih.gov/pmc/articles/PMC4025893/figure/fig01/A, 9B). Chondrocyte apoptosis is thereby demonstrated to be initiated via up-regulation of TRPV5, and this effect can be diminished by TRPV5 inhibition. 
The increase of calcium influx activates calmodulin, which stimulates chondrocyte caspase-8 dependent apoptosis through the activation of FADD

To explore the mechanism whereby upregulation of TRPV5 increases chondrocyte apoptosis, the expression of core proteins was determined by western blotting. As shown in Fig. 10A, 10B, as compared to the untreated group, the expression levels of calmodulin, DAP, FADD, cleaved caspase-8, cleaved caspase-3, cleaved caspase- 6 and cleaved caspase- 7 gradually increase with increasing MIA doses. However, the expression levels of these proteins was decreased in chondrocytes pretreated with ruthenium red and BAPTA-AM or in chondrocytes transfected TRPV5 siRNA correspondingly, compared with the $6 \mu \mathrm{M}$ MIA-stimulated group in Fig. 10C, 10D.

\section{Discussion}

Based on current knowledge, chondrocyte apoptosis could be the underlying factor for the initiation of $\mathrm{OA}$, as well as being involved in the advanced stages of the disease [3]. Understanding the mechanism of chondrocyte apoptosis is essential for developing appropriate targeted therapies for OA treatment. Therefore, a MIA-induced experimental OA rat model was developed to imitate the articular cartilage degeneration, in order to study the mechanism of chondrocyte apoptosis. The advantages of the MIA-induced experimental OA model are that it involves a quick and easy procedure, produces OA-like lesions, and displays functional impairment similar to that observed in the human disease [14].

Since the TRPV family was first discovered in early 1997 [15] and was proposed systematically in 2001 [6], TRPV proteins have been investigated in the

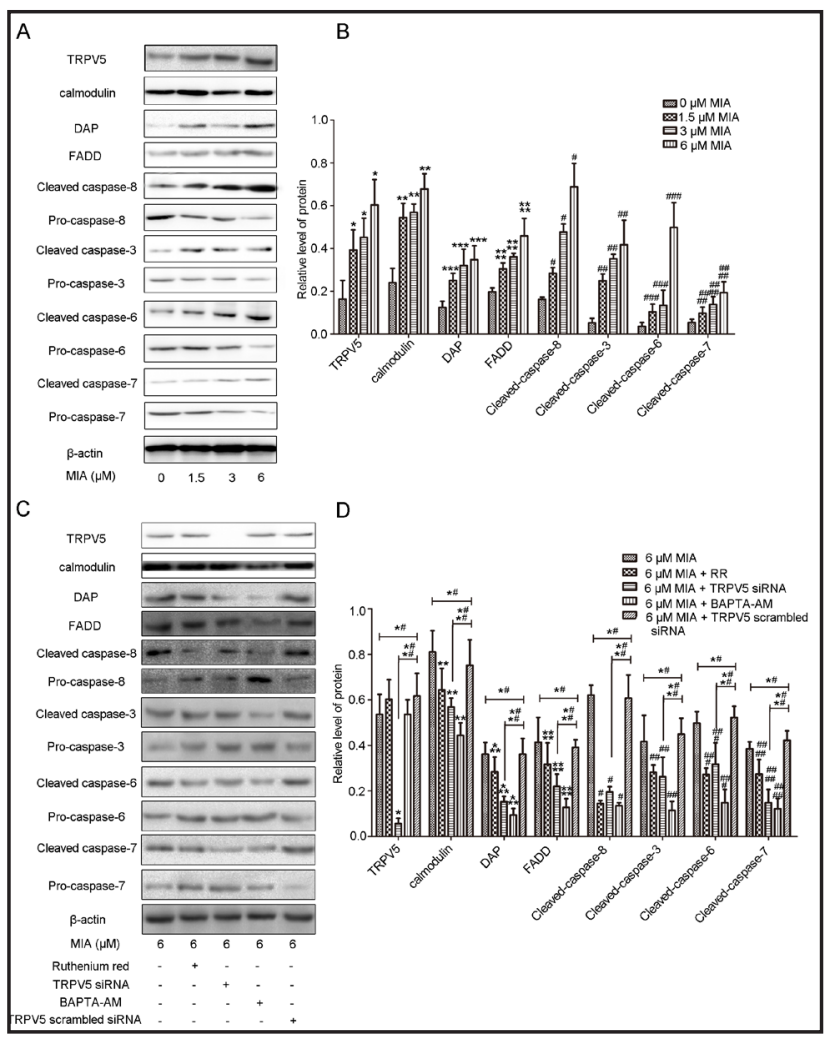

Fig. 10. Increased calcium influx induces chondrocyte apoptosis by activating the TRPV5- $\mathrm{Ca}^{2+}$-calmodulin signaling pathway. (A) TRPV5, calmodulin, DAP, FADD, cleaved caspase-8, cleaved caspase-3, cleaved caspase- 6 , and cleaved caspase-7 protein expression from different MIA concentration groups were detected by western blotting. (B) A bar graph showing relative levels of TRPV5, calmodulin, DAP, FADD, cleaved caspase-8, cleaved caspase-3, cleaved caspase-6, and cleaved caspase-7 protein. ${ }^{*} \mathrm{P}<0.05$; ${ }^{* *} \mathrm{P}<0.05$; ${ }^{* * *} \mathrm{P}<0.05$; ${ }^{* * * *} \mathrm{P}<0.05$; \# $\mathrm{P}<0.05$; \#\# $\mathrm{P}<0.05$; \#\#\# $\mathrm{P}<0.05$; \#\#\# $\mathrm{P}<0.05$ vs. untreated control. (C) TRPV5, calmodulin, DAP, FADD, cleaved caspase-8, cleaved caspase-3, cleaved caspase-6, and cleaved caspase-7 protein expression from different stimulated groups were detected by western blotting. (D) A bar graph showing the levels of TRPV5, calmodulin, DAP, FADD, cleaved caspase-8, cleaved caspase- 3 , cleaved caspase- 6 and cleaved caspase-7 proteins. ${ }^{*} \mathrm{P}<0.05$; ${ }^{* *} \mathrm{P}<0.05 ;{ }^{* * *} \mathrm{P}<0.05$; ${ }^{* * * *} \mathrm{P}<0.05$; ${ }^{*} \mathrm{P}<0.05$; ${ }^{\# \#} \mathrm{P}<0.05$;

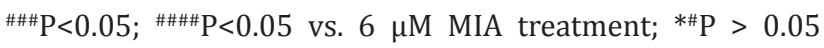
between $6 \mu \mathrm{M}$ MIA and TRPV5 scrambled siRNA chondrocyte + $6 \mu \mathrm{M}$ MIA; ${ }^{* * \# \#} \mathrm{P}<0.05$ between TRPV5 siRNA chondrocyte $+6 \mu \mathrm{M}$ MIA and TRPV5 scrambled siRNA chondrocyte +6 $\mu \mathrm{M}$ MIA. MIA, monosodium iodoacetate; RR, ruthenium red; TRPV5, transient receptor potential channel vanilloid 5; FADD, FAS-associated death domain; DAP, death-associated protein; BAPTA-AM, 1, 2-bis-(o-Aminophenoxy)-ethane$\mathrm{N}, \mathrm{N}, \mathrm{N}^{\prime}, \mathrm{N}^{\prime}$-tetraacetic acid, tetraacetoxymethyl ester. 
etiologies of diseases in many systems. Transient receptor potential channel vanilloid 5 (TRPV5) is a member of the TRPV subfamily that functions as a facilitative $\mathrm{Ca}^{2+}$ transporter. TRPV5 expression has been previously described in tissues involved in $\mathrm{Ca}^{2+}$ transport including hepatocytes, osteoblasts, and enterocytes. TRPV5 has also been found to be expressed in neurons [16] and other non-neurons like smooth muscle cells [17]. It is noteworthy that few studies have reported the functionality of TRPV5 in articular chondrocytes. In our study, we have comprehensively shown that TRPV5 expression in cartilage and up-regulation of TRPV5 participated in the development of OA in the MIA-induced rat model; TRPV5 expression was weak in normal cartilage (sterile saline), while mild staining was observed in moderate severity degenerative cartilage (MIA 7 days, MIA 14 days). High TRPV5 expression was detected

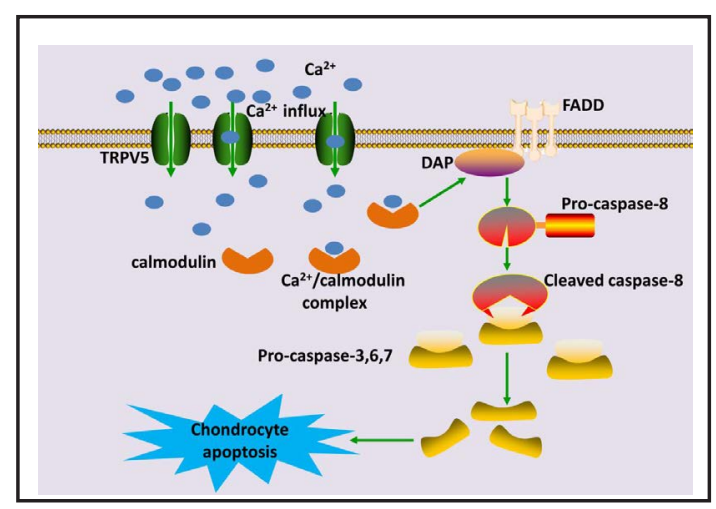

Fig. 11. Diagram of the signaling cascade involved in the effect of TRPV5-mediated $\mathrm{Ca}^{2+}$ influx on chondrocyte apoptosis. TRPV5 may exert a core initiation effect to mediate the induction of apoptosis signaling via cytosolic $\mathrm{Ca}^{2+}$ accumulation. TRPV5, transient receptor potential channel vanilloid 5; FADD, FAS-associated death domain; DAP, deathassociated protein.

in the articular cartilage of the most severe OA (MIA 21 days) (Fig. 4A, 4D). A direct linear correlation was obtained between the TRPV5 expression and the severity of the OA. We also found that calmodulin and the apoptosis marker-cleaved caspase- 8 were significantly upregulated in OA cartilage in a positive linear relationship with TRPV5 protein (Fig. 3B, 3C). We speculate that the expression of calmodulin linked with osteoarthritis may be promoted by TRPV5-mediated $\mathrm{Ca}^{2+}$ influx. It has been previously reported that abnormal TRPV5 can cause $\mathrm{Ca}^{2+}$ influx overloading in HEK293 [18] and mice ear hair cells [19]. Our study delineated that $\mathrm{Ca}^{2+}$ increase via intracellular influx through TRPV5 can inhibit chondrocyte autophagy in OA [5]. The complex role of TRPV5 expression in cartilage chondrocytes may require further investigation.

So, we verified the functionality and activation of the TRPV5 channel in primary rat chondrocytes by measuring $\mathrm{Ca}^{2+}$ influx using a Fluo-4AM. Fluo-4AM Assay Kit, which is one of the commonly used methods to measure free calcium ions in cells [20]. Fluorescence values $(\mathrm{F})$ were normalized by the non-specific background fluorescence $\left(\mathrm{F}_{0}\right)$ to obtain the fluorescence ratio $\left(F / F_{0}\right)$, and the relative fluorescence intensity was counted statistically as percentage variation of treatments from untreated $(0 \mu \mathrm{M}$ MIA). Even if Fluo-4AM is not a radiometric dye, it can also be used to detect changes in relative fluorescence values if there are changes in free calcium concentration. The relative fluorescence intensity in the MIA-stimulated group increased gradually with increasing concentrations of MIA, while the fluorescence intensity was significantly reduced by treating with the TRPV5 specific inhibitor, ruthenium red or BAPTA-AM, or in chondrocytes transfected TRPV5 siRNA. Our results suggested that TRPV5 had a specific effect on cytosolic $\mathrm{Ca}^{2+}$ concentration and on mediating extracellular calcium influx. Indeed, $\mathrm{Ca}^{2+}$ entry pathways have been identified in developing and mature chondrocytes including N-methyl-d-aspartate receptor (NMDA) subunits [21], transient receptor potential (TRP) channels and voltage-operated $\mathrm{Ca}^{2+}$ channels (VOCCs) [22]. Among these pathways, TRPV is an important channel, but has been less studied. The TRPV4 ion channel has been reported as being a key mediator and significant component of chondrocyte mechanotransduction pathways [23]. $\mathrm{Ca}^{2+}$ is a major intracellular second messenger and has a direct or indirect role in mediating apoptosis [4]. $\mathrm{Ca}^{2+}$ was highlighted as being important in apoptosis involving calcium entry-dependent reactive oxygen species (ROS) production [24], mitochondrial depolarization and DNA fragmentation [25]. Although most studies regarding the role of $\mathrm{Ca}^{2+}$ in apoptosis have mainly focused on its increased 


\section{Cellular Physiology Cell Physiol Biochem 2018;46:687-698 \begin{tabular}{l|l} 
DOI: 10.1159/000488725 & O 2018 The Author(s). Published by S. Karger AG, Basel \\
www.karger.com/cpb
\end{tabular} \\ Wei et al.: Transient Receptor Potential Channel, Vanilloid 5, Induces Chondrocyte Apoptosis}

release from the ER, the role of $\mathrm{Ca}^{2+}$ influx through $\mathrm{Ca}^{2+}$-selective channels has also been studied extensively $[5,18,26]$. Our results suggested that calcium influx though the TRPV5 channel could induce apoptosis of chondrocytes.

Mutation of TRPV5 channels in lymphocytes can also activate apoptosis of lymphocytes [27], but the exact mechanism is not clear. In the present study, flow cytometric analysis confirmed that upregulated TRPV5 leads to apoptosis and that both pharmacological inhibition and siRNA-mediated knockdown of TRPV5 can reduce the apoptosis rate of MIAinduced chondrocytes.

The involvement of DAP-kinase in Fas-induced cell death is supported by independent evidence [28]. Knockdown of FADD blocks Fas-induced activation of caspase-8 and caspase-3, thus rendering them resistant to Fas-induced apoptosis [29]. Western blotting indicated that up regulated the TRPV 5 channel can activate calmodulin, DAP, and FADD proteins in this pathway. In turn, activated FADD can stimulate caspase-8, which can activate caspases-3, 6, and 7. The cascade activation pathway was shown in Fig. 9. Our results were consistent with our previous hypothesis (Fig. 11).

In summary, we found that the TRPV5 cation channel was functionally expressed in normal cartilage and upregulated in OA articular cartilage. The up-regulated TRPV5 could be an initiating factor that induces extrinsic chondrocyte apoptosis via the mediation of $\mathrm{Ca}^{2+}$ influx. These findings suggested TRPV5 could be as an intriguing mediator for drug target in $\mathrm{OA}$.

\section{Acknowledgements}

This work was supported by the National Natural Science Foundation of China (General Program; No.: 81272050; No.: 81772420); Excellent PhD Program of ShengJing Hospital of China Medical University (No.: ME332).

\section{Disclosure Statement}

No conflict of interests exists.

\section{References}

1 Cross M, Smith E, Hoy D, Nolte S, Ackerman I, Fransen M, Bridgett L, Williams S, Guillemin F, Hill CL, Laslett LL, Jones G, Cicuttini F, Osborne R, Vos T, Buchbinder R, Woolf A, March L: The global burden of hip and knee osteoarthritis: estimates from the global burden of disease 2010 study. Ann Rheum Dis 2014;73:1323-1330.

2 Wei Y, Bai L: Recent advances in the understanding of molecular mechanisms of cartilage degeneration, synovitis and subchondral bone changes in osteoarthritis. Connect Tissue Res 2016;10.1080/03008207.2016.11770361-17.

-3 Hwang HS, Kim HA: Chondrocyte Apoptosis in the Pathogenesis of Osteoarthritis. Int J Mol Sci 2015;16:26035-26054.

-4 Kim JY, Yu SJ, Oh HJ, Lee JY, Kim Y, Sohn J: Panaxydol induces apoptosis through an increased intracellular calcium level, activation of JNK and p38 MAPK and NADPH oxidase-dependent generation of reactive oxygen species. Apoptosis 2011;16:347-358.

5 Wei Y, Wang Y, Wang Y, Bai L: Transient Receptor Potential Vanilloid 5 Mediates Ca2+ Influx and Inhibits Chondrocyte Autophagy in a Rat Osteoarthritis Model. Cell Physiol Biochem 2017;42:319-332.

6 Montell C: Physiology, phylogeny, and functions of the TRP superfamily of cation channels. Sci STKE 2001;2001:re1.

7 Nijenhuis T, Hoenderop JG, Bindels RJ: TRPV5 and TRPV6 in Ca(2+) (re)absorption: regulating Ca(2+) entry at the gate. Pflugers Arch 2005;451:181-192. 


\section{Cellular Physiology Cell Physiol Biochem 2018;46:687-698 \begin{tabular}{l|l} 
DOI: 10.1159/000488725 & Ond Biochemistry 2018 The Author(s). Published by S. Karger AG, Basel \\
wwww.karger.com/cpb
\end{tabular} \\ Wei et al:: Transient Receptor Potential Channel, Vanilloid 5, Induces Chondrocyte Apoptosis}

8 Dai W, Bai Y, Hebda L, Zhong X, Liu J, Kao J, Duan C: Calcium deficiency-induced and TRP channel-regulated IGF1R-PI3K-Akt signaling regulates abnormal epithelial cell proliferation. Cell Death Differ 2014;21:568-

581.

-9 Chen F, Ouyang Y, Ye T, Ni B, Chen A: Estrogen inhibits RANKL-induced osteoclastic differentiation by increasing the expression of TRPV5 channel. J Cell Biochem 2014;115:651-658.

10 Marshall CB, Nishikawa T, Osawa M, Stathopulos PB, Ikura M: Calmodulin and STIM proteins: Two major calcium sensors in the cytoplasm and endoplasmic reticulum. Biochem Biophys Res Commun 2015;460:521.

11. Papoff G, Trivieri N, Crielesi R, Ruberti F, Marsilio S, Ruberti G: FADD-calmodulin interaction: a novel player in cell cycle regulation. Biochim Biophys Acta 2010;1803:898-911.

12 Adams JM: Ways of dying: multiple pathways to apoptosis. Genes Dev 2003;17:2481-2495.

13 Sun R, Yang Y, Ran X, Yang T: Calcium Influx of Mast Cells Is Inhibited by Aptamers Targeting the First Extracellular Domain of Orai1. PLoS One 2016;11:e0158223.

14 Guingamp C, Gegout-Pottie P, Philippe L, Terlain B, Netter P, Gillet P: Mono-iodoacetate-induced experimental osteoarthritis: a dose-response study of loss of mobility, morphology, and biochemistry. Arthritis Rheum 1997;40:1670-1679.

15 Montell C: New light on TRP and TRPL. Mol Pharmacol 1997;52:755-763.

16. Lang F, Strutz-Seebohm N, Seebohm G, Lang UE: Significance of SGK1 in the regulation of neuronal function. J Physiol 2010;588:3349-3354.

17 Earley S: Vanilloid and melastatin transient receptor potential channels in vascular smooth muscle. Microcirculation 2010;17:237-249.

-18 Lee KP, Nair AV, Grimm C, van Zeeland F, Heller S, Bindels RJ, Hoenderop JG: A helix-breaking mutation in the epithelial $\mathrm{Ca}(2+)$ channel TRPV5 leads to reduced $\mathrm{Ca}(2+)$-dependent inactivation. Cell Calcium 2010;48:275-287.

19 Grimm C, Cuajungco MP, van Aken AF, Schnee M, Jors S, Kros CJ, Ricci AJ, Heller S: A helix-breaking mutation in TRPML3 leads to constitutive activity underlying deafness in the varitint-waddler mouse. Proc Natl Acad Sci U S A 2007;104:19583-19588.

-20 Mohankumar SK, Taylor CG, Zahradka P: Domain-dependent modulation of insulin-induced AS160 phosphorylation and glucose uptake by Ca2+/calmodulin-dependent protein kinase II in L6 myotubes. Cell Signal 2012;24:302-308.

21 Matta C, Zakany R: Calcium signalling in chondrogenesis: implications for cartilage repair. Front Biosci (Schol Ed) 2013;5:305-324.

22 Fodor J, Matta C, Olah T, Juhasz T, Takacs R, Toth A, Dienes B, Csernoch L, Zakany R: Store-operated calcium entry and calcium influx via voltage-operated calcium channels regulate intracellular calcium oscillations in chondrogenic cells. Cell Calcium 2013;54:1-16.

23 Phan MN, Leddy HA, Votta BJ, Kumar S, Levy DS, Lipshutz DB, Lee SH, Liedtke W, Guilak F: Functional characterization of TRPV4 as an osmotically sensitive ion channel in porcine articular chondrocytes. Arthritis Rheum 2009;60:3028-3037.

24 Kovac S, Domijan AM, Walker MC, Abramov AY: Seizure activity results in calcium- and mitochondriaindependent ROS production via NADPH and xanthine oxidase activation. Cell Death Dis 2014;5:e1442.

-25 Llorente-Folch I, Rueda CB, Pardo B, Szabadkai G, Duchen MR, Satrustegui J: The regulation of neuronal mitochondrial metabolism by calcium. J Physiol 2015;593:3447-3462.

-26 Sun Z, Han J, Zhao W, Zhang Y, Wang S, Ye L, Liu T, Zheng L: TRPV1 activation exacerbates hypoxia/ reoxygenation-induced apoptosis in H9C2 cells via calcium overload and mitochondrial dysfunction. Int J Mol Sci 2014;15:18362-18380.

27 Morelli MB, Liberati S, Amantini C, Nabiss M, Santoni M, Farfariello V, Santoni G: Expression and function of the transient receptor potential ion channel family in the hematologic malignancies. Curr Mol Pharmacol 2013;6:137-148.

28 Cohen O, Inbal B, Kissil JL, Raveh T, Berissi H, Spivak-Kroizaman T, Feinstein E, Kimchi A: DAP-kinase participates in TNF-alpha- and Fas-induced apoptosis and its function requires the death domain. J Cell Biol 1999;146:141-148.

-29 Yuan K, Jing G, Chen J, Liu H, Zhang K, Li Y, Wu H, McDonald JM, Chen Y: Calmodulin mediates Fas-induced FADD-independent survival signaling in pancreatic cancer cells via activation of Src-extracellular signalregulated kinase (ERK). J Biol Chem 2011;286:24776-24784. 